ranswase

glyndîn

Glyndŵr University

Glyndŵr University Research Online

Natural Environment

Natural \& Built Environment

6-1-2012

\title{
A novel scale of behavioural indicators of stress for use with domestic horses
}

Tamsin Young

Glyndwr University, t.young@glyndwr.ac.uk

Emma Creighton

Newcastle University

Tessa Smith

Foniprowitłpf_Chester additional works at: http://epubs.glyndwr.ac.uk/nat_env

C. Afthtteftetbesienimals Commons, Large or Food Animal and Equine Medicine Commons, and the

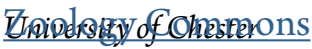

Copyright (C) 2012 Elsevier B.V. All rights reserved.

NOTICE: this is the author's version of a work that was accepted for publication in Applied Animal

Behaviour Science. Changes resulting from the publishing process, such as peer review, editing, corrections, structural formatting, and other quality control mechanisms may not be reflected in this document. Changes may have been made to this work since it was submitted for publication. A definitive version was subsequently published in Young, T. Creighton, E., Smith, T., Hosie C. (2012) "A novel scale of behavioural indicators of stress for use with domestic horses". Applied Animal Behaviour Science, Volume 140 issue 1, pp. 33- 43. DOI:10.1016/j.applanim.2012.05.008 and can be accessed via this link http://dx.doi.org/10.1016/j.applanim.2012.05.008 on the publisher's website at http://www.journals.elsevierhealth.com

\section{Recommended Citation}

Young, T. Creighton, E., Smith, T., Hosie C. (2012) "A novel scale of behavioural indicators of stress for use with domestic horses". Applied Animal Behaviour Science, Volume 140 issue 1, pp. 33- 43.

This Article is brought to you for free and open access by the Natural \& Built Environment at Glyndŵr University Research Online. It has been accepted for inclusion in Natural Environment by an authorized administrator of Glyndŵr University Research Online. For more information, please contact d.jepson@glyndwr.ac.uk. 


\section{A novel scale of behavioural indicators of stress for use with domestic}

horses.

\section{Tamsin Young ${ }^{a,{ }^{*}}$, Emma Creighton ${ }^{b}$, Tessa Smith ${ }^{a}$,} Charlotte Hosie ${ }^{a}$

${ }^{a}$ Department of Biological Sciences, University of Chester, Chester, $\mathrm{CH} 14 \mathrm{BJ}$, UK

${ }^{\mathrm{b}}$ School of Agriculture, Food and Rural Development, Newcastle University, Newcastle, NE1 7RU, UK

*Corresponding author: Tamsin Young, Glyndŵr University, Northop Campus, Mold, Flintshire, CH7 6AA, UK, tel: 07912324308, t.young@glyndwr.ac.uk

\section{Abstract}

Behaviour scores (BS) offer non-invasive, objective and easy to use ways of assessing welfare in animals. Their development has; however, largely focused on behavioural reactions to stressful events (often induced), and little use of physiological measures has been made to underpin and validate the behavioural measures. This study aimed to develop a physiologically validated scale of behavioural indicators of stress for the purpose of welfare assessment in stabled domestic horses. To achieve this, behavioural and physiological data were collected from 32 horses that underwent routine husbandry procedures. Principal component analysis (PCA) of the behavioural and physiological data revealed three meaningful components that were used as the basis of the scale. Analysis of video clips of the horses' responses to the husbandry procedures was undertaken by a panel of equestrian industry professionals using a free choice profiling (FCP) methodology. These results were added to the scale along with key definitions from relevant literature. Salivary cortisol levels were significantly correlated with the BS confirming 
the scale was meaningful and reflected physiological stress. The scale offers an easy to use 'tool' for rapid, reliable non-invasive welfare assessment in horses, and reduces the need for potentially invasive physiological measures.

\section{Key words}

Horse; behaviour scores; cortisol; saliva; welfare assessment; non-invasive.

\subsection{Introduction}

Use of behaviour scores (BS) offers objective, immediate methods of welfare assessment in animals (Minka et al., 2009). They have been used, with varying degrees of success, to measure stress levels for the purpose of welfare assessment in various species (horses: Visser et al., 2010; Munsters et al., 2011; cats: McCune, 1994; Kessler and Turner, 1997; McCobb et al., 2005; Dybdall et al., 2007; goats:

Minka et al., 2009; cattle: Maria et al., 2004b; ostriches: Minka and Ayo, 2008; chickens: Maria et al., 2004a). These scores have, however, been largely developed by focusing on expression of behaviours assumed to indicate stress rather than making use of established physiological indicators of stress to underpin them. In addition, BS are often used rather simply, ignoring all but 'negative' behaviours (e.g. Minka et al., 2009) and therefore losing potentially valuable information. Of the small number of behaviour scoring approaches to welfare assessment available, only the Cat Stress Score (McCune, 1994; Kessler and Turner, 1997) provides a scale of stress responses that can be used flexibly to assess welfare in different environments. This scale has not been physiologically validated, however.

Assessment of a stress response is clearly best carried out using a combination of both behavioural and physiological measures (Broom, 1991; Mason and Mendl, 1993; Dawkins, 2003). This provides a more comprehensive measurement of stress, and avoids drawing misleading conclusions that could be reached by taking just a 

2

single measure (Broom, 1991; Mason and Mendl, 1993; Dawkins, 2003). Previous success at correlating behavioural measures with physiological measures has, however, been mixed. Minka et al. (2009) established that certain behaviours and physiological indices of stress were related during the handling and loading of goats for transportation, but McCobb et al. (2005) were not able to correlate BS with urinary cortisol measures in cats. Clearly the behavioural and physiological measures must both be sufficiently sensitive and reliable to successfully correlate them for use in a behavioural scale for welfare assessment.

The only scale of BS available for use with domestic horses was developed to assess, specifically, whether horse and rider combinations were appropriate (Munsters et al., 2011). The scale was adapted from a scoring system used by Visser et al. (2010) to assess the temperaments of sports horses exposed to novel objects, not to evaluate stress responses. Some level of experience/expertise was needed to accurately assess temperament in this latter study. Physiological measures were not used to develop the score, although heart rate variability (HRV) was used when the score was tested.

The aim of the current study was, therefore, to develop an easy to use scale of BS that could be used to rapidly but reliably assess stress levels in domestic stabled horses. The scale was developed using both behavioural and physiological measures obtained from a wide range of horses $(\mathrm{N}=32)$ during naturally occurring daily routine husbandry procedures. Use of such husbandry procedures, that would have taken place irrespective of the study, was considered an ethical approach to data collection rather than artificially exposing horses to potentially stressful

81 situations 
83 Since assessment of welfare is most effective using multiple measures, analysis of

84 the behavioural responses to the routine husbandry procedures used two different

85 approaches. Behavioural responses were quantified in detail using The Observer

86 (Noldus Information Technology Software Ltd) for 12 of the horses. Both quantitative

87 and qualitative assessment of the behaviour of all study horses was made, by expert

88 panel members, using the experimental free choice profiling (FCP) approach

89 (Wemelsfelder et al., 2000; 2001).

90

91 We again used two measures for the physiological data collection, heart rate (HR)

92 and salivary cortisol, to validate the BS scale. Both cortisol (Ralston et al., 1988;

93 Toutain et al., 1995; McBride and Cuddeford, 2001; Covalesky et al., 1992;

94 Stegaman and Jones, 1998) and HR (Reitmann et al., 2004; von Borell et al., 2007;

95 Visser et al., 2008) are established indicators of stress in horses, and detect different

96 aspects of the stress response. Both indices of stress were used here as their

97 measurement can be achieved by non-invasive means, thus avoiding any further

98 stress to the study horses.

99

100

2.0 Method

$101 \quad 2.1$ Subjects used for the study

102 The study aimed to ensure a wide range of horses was used to build the BS scale.

103 The horses used in the study consisted of various breeds of stabled mares and

104 geldings kept in similar management and exercise regimes, at four different locations

105 (Table 1). All horses were housed in individual stables on either straw or shavings

106 bedding, and received hay or haylage and water with up to two hard feeds i.e. mix or

107 pellets, at around 07:00 $\mathrm{h}$ and 16:00 h. All horses were in light to medium work

108 (receiving around two hours of exercise daily) throughout the study. When they were

109 not being exercised all horses received up to four hours turn out to pasture daily, and 
110 remained in their usual daily management routine apart from undergoing routine

111 husbandry procedures.

112

113 A total of 32 horses were used in this study and assigned to experimental groups on

114 the basis of their availability (see Table 1 for details of location, age, gender and

115 husbandry procedure). In summary, analysis of behaviour was completed from all 32

116 horses; nineteen of the horses were used for saliva collection for cortisol

117 measurement, with a further 10 horses from location one used as a control group for

118 this part of the study; eighteen horses were used for measurement of HR, with a

119 further 10 horses from location one again used as a separate control group for this

120 aspect of the study.

121

\section{$122 \quad 2.2$ Husbandry procedures used for data collection}

123 Horses were subjected individually to one of four different 10 minute husbandry

124 procedures. They remained loose in their stables, except during grooming when they

125 were loosely held by a familiar handler and had access to hay and water. A 10

126 minute period was deemed adequate to induce and measure a potential stress

127 response, but not so long that habituation to the stressor should occur (Visser et al.,

128 2001).

130 Procedure 1 - sound of electric coat clippers

131 Horses (from location 1 only, for logistical reasons) were exposed to the sound of

132 electric coat clippers (Heiniger Handy Clipper, Switzerland) turned on to maximum

133 clipping velocity. Typical sound emissions from such clippers were 80.1 decibels.

134 Clippers were switched on and held by hand in an adjacent stable 3.66 metres away

135 from the study horse.

136

137 Procedure 2 - social isolation 
138 Horses (from locations 1, 2, 3) were caught from the field and returned to their usual

139 stable. This process took no longer than five minutes, and horses showed no

140 resistance to capture. The horses were stabled in the absence of any other horses

141 on the yard for 10 minutes. At the end of the social isolation period the horse's usual

142 neighbouring horse was returned to the adjacent stable.

\section{Procedure 3 - grooming procedures}

145 A head-collar and lead rope was fitted to the horse, and a familiar handler held the

146 lead rope approximately half way along its length to restrain the horse loosely. Mane

147 combing and mane pulling (a procedure used to thin and shorten the mane by taking

148 small sections of hair back combing them and pulling out the remaining long hairs)

149 then took place. Animals were used from location 1.

150

151 Procedure 4 - the sound of fireworks played on a CD

152 Police horses (from location 4 only) were used for this procedure as it involved the

153 sound of fireworks played on a compact disc (CD), which was used as part of riot

154 training with Police horses. The CD player was situated on a table outside the

155 horse's stable 3.66 metres away.

156

157 The husbandry procedures were carried out over a number of weeks as the

158 opportunities to collect data arose. Where the same procedures were carried out

159 with a number of horses on the same yard e.g. exposure to the sound of electric coat

160 clippers, one week was left between tests to minimise the effects of habituation on

161 the horses that had not yet been sampled from. The control horses were chosen

162 randomly from location one, as these had not been subjected to the husbandry

163 procedures examined in the study for a minimum of eight weeks. This opportunistic

164 data collection strategy meant that some procedures/control were tested in a single 
165 location, others across more than one. Location effects were tested for in the

166 analysis to check that this had no confounding impact.

\subsection{Behavioural measurement during the husbandry procedures}

169 The behaviour of the subjects was recorded during all husbandry procedures using a

170 Sanyo CCD/BW video camera (Sanyo Electric Co., Ltd, Osaka, Japan) secured at

171 ceiling height in an appropriate position opposite the stable to gain an adequate field

172 of view. The video camera was linked to a Mitsubishi HS-1024E time-lapse recorder

173 (Osaka, Japan), set to three hour real time for recording of images onto three hour

174 video tapes (BASF Vision Chrome Videocassette, BASF plc, Middlesex, U.K.).

\subsubsection{Analysis of behaviour using The Observer}

177 The first five minutes of the behavioural reactions exhibited during the husbandry

178 procedures, for 12 of the horses, was analysed using The Observer 5.0. Behaviours

179 were recorded using a pre-defined ethogram based on equine stable behaviour

180 (Table 2). The ethogram had been compiled from six weeks of ad-hoc observation of

181 race-horses and stabled riding horses, together with literature research (see Houpt,

182 1993; Winskill et al., 1996; McBride and Cuddeford, 2001; Strand et al., 2002;

183 Heleski et al., 2002; Seaman et al., 2002; McDonnell, 2003)

184

$185 \quad$ 2.3.2 Analysis of behaviour carried out by a panel of equestrian professionals

186 A panel of 13 professionals who had a working background with horses, and held a

187 minimum of the British Horse Society (BHS) stage one qualification was convened.

188 They were briefed on the nature of the study and asked to view a video of the initial

189 two minutes of each horse's behavioural reaction to the husbandry procedures, and

190 to provide a BS between zero and ten according to how stressed they perceived the

191 horse to be. They were told that ten equated to an extremely stressed horse. The

192 panel also had to describe, using their own descriptive terms, the horse's behaviour 
193 exhibited during the video. They, finally, were asked to state at which point on their

194 subjective scale that they believed the onset of stress occurred in the horses

195 undergoing the husbandry procedures.

196

\subsection{Measurement of salivary cortisol concentrations during the husbandry} procedures

199 Saliva was collected 60 minutes and 30 minutes prior to the start of the husbandry

200 procedures, and then at the end of the 10 minute procedure and at 10 minute

201 intervals up to 40 minutes. Forty minutes was chosen to provide enough time for

202 peak cortisol to be reached following the onset of the potential stressor (the

203 husbandry procedure). Plasma cortisol peaks in horses 30 minutes post exercise

204 stress (Foreman and Ferlazzo, 1996; Marc et al., 2000; Hamlin et al., 2000), and 20

205 minutes following restraint stress (Hydbring et al., 1996).

207 Saliva was collected from the control group of horses to ensure that the swabbing 208 procedure did not affect their stress levels. Collection took place at 60 minutes, 30 209 minutes, and 0 minutes before the husbandry procedure would have begun, and then

210 at the same time intervals that the experimental group of horses had their saliva

211 collected, except for the extra swab was taken at 0 minutes from control horses to 212 provide a robust control measure.

214 Saliva was collected using sterilised flexi-swabs (Medical Wire \& Equipment Co

215 (Bath) Ltd) that were introduced into the corner of the horses' mouths first on the 216 horse's left and then on the horse's right. The horses were allowed to manipulate the 217 swabs using their tongues for approximately 20 seconds per introduction of the swab.

218 The swabs were then placed into sterile $20 \mathrm{ml}$ plastic screw top containers, labelled

219 and stored on ice until frozen at $-20^{\circ} \mathrm{C}$ the same day to await cortisol extraction. 
221 Saliva was extracted from the thawed cotton wool swabs by centrifugation using a

222 Sorvall T.C. centrifuge (Thermo Scientific, Basingstoke, Hampshire, UK) for two 223 minutes at $800 \mathrm{~g}$. The supernatant was then centrifuged using a Hettick Mikro 20 224 centrifuge (Tuttilgen, Germany) at 15,000g for two minutes. The supernatant was

225 taken off using a pipette and frozen to await analysis. Salivary cortisol

226 concentrations were quantified using a modified version of an EIA described by

227 Smith and French (1997).

\subsection{Measurement of heart rate (HR) during the husbandry procedures}

$230 \mathrm{HR}$ was recorded from the experimental and control groups of horses at 60-second 231 intervals for two minutes prior to the start of the husbandry procedure to provide a 232 mean baseline HR. Recording of HR then continued at 60 -second intervals for the 233 first five minutes of the husbandry procedure for the experimental group of horses, 234 and over the same time intervals, in the absence of a husbandry procedure, for the 235 control group.

237 HR was recorded using a Polar HR monitor (S610i) (Polar Electro, Öy, Kempele, 238 Finland). The HR monitor consisted of an electrode belt that picked up the electrical 239 activity of the horse's heart, with a transmitter attached enabling wireless 240 transmission of the HR to a wrist watch receiver. The belt was fitted around the 241 horse's thorax with both electrodes sited to the left-hand side of the horse. One 242 electrode was placed approximately $10 \mathrm{~cm}$ below the withers, and the other about $24310 \mathrm{~cm}$ behind the elbow over the heart. Warm water and electrode gel (The Wyke of 244 Shifnal, Shropshire) was used to optimise contact between the horse's skin and the 245 electrodes. The wrist watch receiver was taped to a leather strap fastened around 246 the horse's neck. All horses were given 10 minutes to habituate to the equipment 247 (Reitmann et al., 2004). 


\subsection{Statistical analysis}

250 Principal component analysis (PCA) was used to investigate whether there were any 251 relationships between behavioural and physiological changes that took place during 252 the husbandry procedures. 'Data reduction' was necessary to look for smaller sets of 253 factors or components in the data (Pallant, 2004; Ennos, 2007) from which the scale 254 of BS could be compiled. The percentage change in cortisol concentration from the 255 median baseline value to the peak concentration was calculated for each horse. This 256 percentage, together with the percentage duration of all behaviours included in the 257 ethogram underwent PCA.

PCA of the cortisol and behavioural data exhibited during the husbandry procedures revealed correlation coefficients of 0.3 and above (following Pallant, 2004). An

261 oblimin rotation of three factor solution was used to reduce the number of variables 262 into meaningful components (Pallant, 2004). Each behaviour and change in cortisol 263 concentration received a score for each component denoting whether the behaviour 264 was performed or not, or whether change in cortisol was relevant. A median BS was 265 calculated for each horse used in the study, as scored by members of the 266 professional panel. The terms used by the panel to describe each horse's behaviour 267 was pooled for horses with the same BS. Panel descriptions of behaviour were 268 added to the relevant sets of factors or components revealed by the PCA, and the 269 scale of BS for use with stabled domestic horses subsequently devised.

271 All HR and salivary cortisol data measured during the husbandry procedures and 272 controls were log transformed to reduce heterogeneity of variance. The impact of 273 location on each physiological measure was investigated to assess potential 274 confounds caused by using subjects at different locations. To do this, levels of 275 baseline cortisol and HR data were compared across the two locations in the case of 
276 HR data using an independent samples T test, and across the four locations in the

277 case of salivary cortisol data using a one-way between subjects ANOVA.

279 Any impact of the Polar heart monitor over time was explored during a control study 280 by analysing HR at time zero and $60,120,180,240$ and 300 seconds as compared 281 to the experimental timings, under control conditions. The impact of collecting saliva 282 swabs on the HPA response was examined during a control study which assessed 283 cortisol under control conditions at time 0 , then 10, 20, 30 and 40 minutes later using 284 a repeated subjects ANOVA.

286 Changes in HR data and levels of salivary cortisol were explored using General 287 Linear Models (GLM) with sex, husbandry procedure and time of collection (i.e. 288 before and after the procedure in terms of the HR data, and peak compared to 289 baseline cortisol titres for the salivary cortisol data) as fixed factors. Post hoc 290 analyses were conducted using Tukey test and alpha was set at 0.05.

292 To investigate whether the devised BS scale reflected physiological stress, median 293 BS as calculated from the professional panel, and peak salivary cortisol following the husbandry procedures were investigated using Spearman's Rank Order Correlation.

\subsection{Results}

\section{$297 \quad 3.1$ Behavioural data}

298 PCA of the percentage change in salivary cortisol from baseline to peak, and 299 percentage duration of state behaviour exhibited during the husbandry procedures 300 identified three components in the pattern matrix. They were labelled no stress

301 (factor 1), low stress (factor 3 ) and medium stress (factor 2) according to the type of 302 behaviour and change in cortisol identified (Table 3). 
304 Median behaviour scores were calculated for the study horses, and ranged between

305 one and eight. The terms used by the panel to describe each horse's behaviour was

306 pooled for horses with the same BS, and panel descriptions of behaviour were added

307 to the three components revealed by the PCA.

309 Descriptions used for horses with a BS of one and two were added to the component

310 labelled no stress, as the mean score representing the onset of stress as judged by

311 the panel was three. Descriptions used for horses with a BS of three to seven were

312 added to low and medium stress. The BS of five was used as the onset of medium

313 stress, based on the type of behaviour included in the component extracted by the

314 PCA. Descriptions used for horses with a BS of eight to ten formed a new category

315 labelled high stress, because the PCA analysis did not include horses with a BS of

316 this level. Relevant literature was also used to form this category. The scale of

317 behavioural indicators of stress for use with stabled domestic horses was

318 subsequently compiled (Table 4).

\subsection{Physiological data}

321 Both measures of HR and salivary cortisol were used to underpin the behavioural measures in the development of the scale of behavioural scores.

\subsubsection{HR data}

325 Baseline HR values were comparable across the two locations $(t=-0.660, d f=16$,

326 NS) thus ruling out location as a confound in the study. There were, also, no

327 significant changes in HR between baseline (mean HR $37.20 \pm 8.34 \mathrm{bpm}$ ) and the

328 'test' period (mean HR $38.98 \pm 15.65 \mathrm{bpm}$ ) in the control study suggesting that the

329 presence of the Polar heart rate monitor over a period of time did not cause the

330 horses any stress $(t=-0.381, d f=9, N S)$. 
332 HR values were explored using a general linear model with sex, husbandry

333 procedure and time of collection (i.e. before versus after the procedure) as the fixed

334 factors. HR values were significantly raised following the husbandry procedures

335 compared to baseline values (Figure 1) $[\mathrm{F}(1,26)=10.083, \mathrm{P}<0.0001]$.

337 There was no effect of sex on $\operatorname{HR}$ values $[F(1,26)=0.261, N S]$, but there was a

338 significant interaction between sex and husbandry procedure $[F(1,26)=4.315, P<$

339 0.05]. Further analysis using a t test showed that $\mathrm{HR}$ values before and after

340 combined, for the clippers, were significantly higher for mares than the same values

341 for geldings $(\mathrm{t}=-3.403, \mathrm{df}=18, \mathrm{P}<0.003)$. In contrast for grooming, before and after

342 HR values (combined) were similar for both mares and geldings $(t=1.294, d f=6$,

343 NS). Only geldings were exposed to the fireworks.

345 There was no effect of husbandry procedure alone on $\operatorname{HR}$ values $[F(2,26)=2.444$,

$346 \mathrm{NS}]$, or interaction between husbandry procedure and sampling time $[\mathrm{F}(2,26)=$

$3470.621, N S]$, sex and sampling time $[F(2,26)=0.169, N S]$, or between the three

348 variables $[\mathrm{F}(1,26)=0.820, \mathrm{NS}]$.

\section{$350 \quad 3.2 .2$ Salivary cortisol data}

351 Baseline salivary cortisol titres levels were comparable across subjects in the four

352 locations confirming that location was not a confound in our study $[F(3,18)=1.824$, 353 NS].

355 Changes in physiological data collected from control horses were explored to ensure 356 that the methods of data collection did not affect the horses. There were no changes 357 in levels of salivary cortisol across the control study (mean \pm s.e.m.) baseline $0.42 \pm$ $3580.12 \mathrm{ng} / \mathrm{ml}$; at 10 minutes $0.43 \pm 0.2$; at 20 minutes $0.35 \pm 0.17$; at 30 minutes $0.39 \pm$ 
0.15 ; at 40 minutes $0.49 \pm 0.25)$ suggesting that the saliva swabbing was not

360 stressful to our subjects $[\mathrm{F}(4,32)=0.821, \mathrm{NS}]$.

362 There was a significant effect of husbandry procedure on levels of salivary cortisol [F $363(3,22)=5.644, P<0.005]$. Post hoc analysis revealed that levels of the hormone 364 (baseline and peak concentrations combined) pertaining to exposure to the fireworks 365 were significantly higher than those related to clipping $(P<0.01)$ and social isolation $366(P<0.01)$, but not grooming $(N S)$. Hormone values relating to grooming were higher 367 than those relating to clipping $(P<0.05)$ and social isolation $(P<0.01)$.

There was also a significant effect of time (i.e. baseline cortisol versus peak concentration of cortisol) since hormone levels were significantly elevated following the husbandry procedures compared to prior to the procedures (Figure 2) $[F(1,22)=$ $6.077, \mathrm{P}<0.05]$. There was, however, no interaction between husbandry procedure

373 and sample time showing that despite gross differences in hormone values across

374 the different procedures, the manner and magnitude of the change in cortisol levels

375 pre and post treatment were comparable across the four husbandry procedures [F (3,

$37622)=0.827$, NS]. This was confirmed by two separate one-way ANOVAs which 377 revealed comparable hormone levels across the husbandry procedures before [F ( 3 , $37814)=3.035, N S]$ and after $[F(3,14)=2.292$, NS] the treatments. There was no 379 effect of sex on hormone values $[F(1,22)=0.645$, NS], and no interaction effects, 380 i.e. condition and $\operatorname{sex}[F(2,22)=2.184, N S]$, sex and time $[F(1,22)=0.000, N S]$, 381 condition, sex and time $[\mathrm{F}(2,22)=0.701, \mathrm{NS}]$.

\section{$383 \quad 3.4$ Correlating behavioural and physiological data}

384 To ensure the final BS scale reflected increased levels of both behavioural and 385 physiological stress, their relationship in response to the husbandry procedures was 
386 investigated. A significant correlation existed between median BS and peak salivary

387 cortisol concentration measured during the husbandry procedures $\left(r_{s}=0.54, P=\right.$

$3880.02, n=18)$ confirming that the final BS scale was a reflection of both behavioural

389 and physiological stress.

\subsection{Discussion}

392 In this study a scale of BS has been developed to measure stress levels in stabled

393 domestic horses for the purposes of welfare assessment. To do this, behaviour and

394 physiology were each measured by two different techniques in a range of horses

395 undergoing standard husbandry procedures. The physiological data were used to

396 underpin behavioural measures. Physiological data revealed that husbandry

397 procedures did significantly elevate HR. HR was greater for mares than geldings for

398 the clipping procedure but overall patterns of change in HR before and after

399 procedures was the same for both sexes. This shows that the husbandry procedures

400 used here were perceived as stressful by the horses, and provides a biological

401 validation of these procedures as stressful events. These data also demonstrate the

402 sensitivity of HR as a reliable physiological measure of stress.

403

404 Similarly, cortisol measures demonstrated that the husbandry procedures had a

405 significant effect on stress physiology. Importantly these data also showed that

406 location (source of horses), sex and other factors did not affect cortisol measures.

407 Again, this provides biological validation of these procedures as stressful events and 408 demonstrates that salivary cortisol is a sensitive, reliable measure of stress. These

409 two physiological measures can, therefore, be considered reliable to use to underpin

410 the development of the scale of behavioural scores. Schmidt et al. (2010) recently

411 also established use of HR and salivary cortisol together as sensitive parameters to

412 detect stress in 'routine' transport procedures for horses. 
414 Behavioural data (percentage durations) from each horse during the husbandry

415 procedures was combined with percentage change in cortisol from baseline to peak

416 in PCA analysis. The three components revealed by the PCA were combined with

417 the expert panel descriptions to derive the scale of behavioural indicators of stress

418 that make up the behavioural stress score this work has developed. To ensure the

419 final scale of BS reflected increasing levels of behavioural and physiological stress,

420 the relationship between behavioural and physiological changes in response to the

421 husbandry procedures was investigated. Measures of salivary cortisol concentration

422 following the routine husbandry procedures, and the median BS calculated for the

423 same animal were seen to correlate. In doing this we have successfully combined

424 two different behavioural approaches, underpinning them with two well-established

425 physiological measures.

427 Whilst other studies have used BS these are often relatively simple measures of

428 behaviours. For example Minka et al. (2009) use a number of 'negative' behaviours

429 as a measure of stress, and Maria et al. (2004a) use a greater complexity of

430 behaviours as an indication of less stress. In addition, these may involve a degree of

431 subjectivity in their use (Minka et al., 2009; Munsters et al., 2011) or require

432 sophisticated analysis to assess (Maria et al. 2004a). The BS indicators in our scale

433 developed here are straightforward and do not require great experience of horses to

434 be easily used. In addition, the behavioural descriptions provide a simple scale to

435 enable a range of levels of stress to be measured. Of the small number of behaviour

436 scoring approaches to welfare assessment published, only the Cat Stress Score

437 (McCune, 1994; Kessler and Turner, 1997) provides a sliding scale of stress

438 responses that can be used flexibly to assess welfare in different environments, as

439 used by McCobb et al.( 2005) and Dybdall et al. (2007). This latter scale however,

440 has not been physiologically validated, and McCobb et al. (2005) were not able in

441 their study to correlate BS with urinary cortisol measures in cats housed in traditional 
or enriched shelter environments. We believe our comprehensive approach to

443 combining behaviour and physiology has resulted in a non-invasive, user-friendly,

444 physiologically validated, behavioural stress score that can be used in a variety of

445 environments to measure stress and assess welfare in domestic horses.

447 The work undertaken in developing this behavioural stress score also sheds

448 interesting light on the role of stereotypies in assessing stress in horses. Abnormal

449 or stereotypic behaviour was included in all components extracted by the PCA. 'No

450 stress' horses showed an association with repetitive oral behaviour such as crib-

451 biting, low stress horses exhibited weaving, and both low and medium stress horses

452 carried out repetitive head movements such as head shaking or nodding. It has been

453 suggested that performance of stereotypic behaviour may serve as a way of reducing

454 stress levels, or as a way of horses' providing themselves with some sort of control

455 over their environment (Cooper and Albentosa, 2005). This may explain the fact that

456 horses perceived as experiencing no or low stress were exhibiting stereotypies.

457 Weaving, which is indicative of chronic frustration in horses usually associated with

458 attempts to gain social contact with other horses (Visser et al., 2008), was evident in

459 medium stressed horses. This together with repetitive head movements suggests an

460 increased level of frustration experienced by the horses in the low and medium stress

461 groups. These findings suggest that stress assessment that takes a comprehensive

462 view of all behaviour exhibited, rather than a focus on 'negative' behaviours,

463 including stereotypies, is desirable. The behavioural stress score developed here

464 now makes this possible for domestic horses.

465

$466 \quad 5.0$ Conclusion

467 The scale of behavioural indicators of stress developed in this study provides a quick

468 and easy, reliable method of measuring the stress levels of domestic stabled horses

469 to assess their welfare. It was developed using both behavioural and physiological 
471 reliable indices of physiological change in response to stress. The relationship

472 between behavioural and physiological changes inferred in the scale was further

473 confirmed by the correlation seen between change in salivary cortisol and the same

474 horse's behavioural score. This, therefore, reduces the need to measure various

475 physiological parameters separately to validate the use of the scale and so makes it

476 a valuable, cost-effective tool that could be used by horse owners and behavioural

477 scientists alike.

478

479

480

482

483

484

\subsection{Acknowledgements}

We are grateful to the horse owners for allowing us to collect behavioural and physiological data from their animals. We also appreciated the support given to us by the stable staff at Welsh college of Horticulture, Granary Livery, Belle Vue Livery, and Merseyside Mounted Police unit, and by undergraduate students from the University of Chester who helped with data collection during the study. We also thank members of the professional panel who gave up their time to contribute to this study. Thanks are also extended to Dr. Rob Coleman for his help with the cortisol assay, and to Dr. Joanna Hockenhull for her assistance with this study.

\subsection{References}

Broom, D.M., (1991) Animal Welfare: Concepts and Measurement. Journal of Animal Science 20, 5-19.

Cooper, J.J., Albentosa, M.J., (2005) Behaviour adaptation in the domestic horse: potential role of apparently abnormal responses including stereotypic behaviour. Livestock Production Science. 92, 177-182. 
497 Covalesky, M.E, Russoniello, C.R., Malinowski, K., (1992) Effects of show-jumping

498 performance stress on plasma cortisol and lactate concentrations and heart rate and

499 behaviour in horses. Journal of Equine Veterinary Science. Vol. 12(4), 244-250.

501 Dawkins, M.S., (2003) Behaviour as a tool in an assessment of animal welfare.

502 Zoology. 106, 383-387.

503

504 Dybdall, K., Strasser, R., Katz, T., (2007) Behaviour differences between owner

505 surrender and stray domestic cats after entering an animal shelter. Applied Animal

506 Behaviour Science. 104, 85-94.

507

508 Ennos, R., (2007) Statistical and data handling skills in biology. Second Edition.

509 Harlow: Pearson Education Limited.

510

511 Foreman, J.H., Ferlazzo, A. (1996) Physiological responses to stress in the horse.

512 Pferdeheilkunde 12(4), 401-404.

513

514 Hamlin, M.J., Shearman, J.P., Hopkins, W. G., (2002) Changes in physiological

515 parameters in overtrained standardbred racehorses. Equine Veterinary Journal.

516 34(4), 383-388.

517

518 Heleski, C.R., Shelle, A.C, Nielson, B.D., Zanella, A.J., (2002) Influence on housing

519 on weaning horse behaviour and subsequent welfare. Applied Animal Behaviour

520 Science. 78, 291-302.

522 Houpt, K.A., (1993) Equine stereotypies. The Compendium. Vol. 15(9), 1265-1271. 
524 Hydbring, E., Nyman,S., Dahlborn,K., (1996) Changes in plasma cortisol, plasma

525 behaviour endorphin, heart rate, haematocrit and plasma protein concentration in

526 horses during restraint and use of a naso-gastric tube. Pferdeheilkunde. 12(4), 423-

527427.

529 Kessler, M.R., Turner, D.C., (1997) Stress and adaptation of cats (Felis Silvestris)

530 housed singly, in pairs and in groups in boarding catteries. Animal Welfare. 6, 243-

531254.

532

533 Mal, M.W., Friend, T.H, Lay, D.C, Vogelsang, S.G., Jenkins, O.C., (1991)

534 Behavioural responses of mares to short-term confinement \& social isolation.

535 Applied Animal Behaviour Science. 31, 13-24.

536

537 Marc, M, Parvizi, N, Elendorff, F, Kallweit, E., Elsaesser, F., (2000) Plasma cortisol \&

538 ACTH concentrations in the warmblood horse in response to a standardized treadmill

539 exercise test as physiological markers for evaluation of training status. Journal of

$540 \quad$ Animal Science. 78, 1936-1946.

541

542 Maria, G.A., Escos, J., Alados, C.L., (2004a) Complexity of behavioural sequences

543 and their relationship to stress conditions in chickens (Gallus gallus domesticus): a

544 non-invasive technique to evaluate animal welfare. Applied Animal Behaviour

545 Science. 86, 93-104.

546

547 Maria, G.A., Villarroel, M., Chacon, G., Gebresenbet, G., (2004) Scoring system for

548 evaluating the stress to cattle of commercial loading and unloading. Veterinary

549 Record. 154, 818-821. 
551 Mason, G.J., (1991) Stereotypies and suffering. Behavioural Processes. 25, 103-

552115.

554 Mason, G., Mendl, M., (1993) Why is there no simple way of measuring animal 555 welfare? Animal Welfare. 2(4), 301-319.

557 McBride, S.D., Cuddeford, D., (2001) The Putative welfare - reducing effects of 558 preventing equine stereotypic behaviour. Animal Welfare. 10, $173-189$.

560 McCobb, E., Patronek, G., Marder, A., Dinnage, J., Stone, M., (2005) Assessment of 561 stress levels among cats in four animal shelters. Journal Animal Veterinary Medicine 562 Association. 226, 548-555.

564 McCune, S. (1994) Caged cats: avoiding problems and providing solutions.

565 Newsletter of the Companion Animal Study Group. No. 7.

567 McDonnell, S.M., Haviland, J.C.S. (1995) Agonistic ethogram of the equid bachelor 568 band. Applied Animal Behaviour Science. 43, 147-188.

570 McDonnell, S.M., Freeman, D.A., Cymbalaluk, N.F., Scott, H.C., Hinchcliffe, K., Kyle, 571 B., (1999) Behaviour of stabled horses provided continuous or intermittent access to 572 drinking water. American Journal Veterinary Research. Vol. 60(11) 1451-1456.

574 McDonnell, S., (2003) A practical field guide to horse behaviour. Hong Kong: Eclipse 575 Press.

577 Minka, N.S., Ayo, J.O., Sackey, A.K.B., Adelaiye, A.B., (2009) Assessment and 578 scoring of stresses imposed on goats during handling, loading, road transportation 
579 and unloading, and the effect of pre-treatment with ascorbic acid. Livestock Science.

$580 \quad 125,275-282$.

582 Minka, N.S., Ayo, J.O., (2008) Assessment of the stresses imposed on adult

583 ostriches (Senahio camelus) during handling, loading, transportation and unloading.

584 Veterinary Record. 162-846-851.

585

586 Munsters, C.C.B.M., Visser, K.E.K., van der Broek, J., van Oldruitenborgh-

587 Oosterbaan, M.M.S., (2011) The influence of challenging objects and horse-rider

588 matching on heart rate, heart rate variability and behaviour score in riding horses.

589 The Veterinary Journal. (In press).

590

591 Pallant, J., (2004) SPSS Survival Manual. A step by step guide to using SPSS.

592 Maidenhead: Open University Press.

593

594 Ralston, J.M., Stenhouse, A.M., Stenhouse, N.S., Buck, G.J., Lucks, S.F.,

595 Reynoldson, J.A., Bolton, J.R., (1988) Cortisol concentrations in blood and urine of

596 horses. Australian Veterinary Journal. Vol. 65(1), 1-5.

597

598 Rietmann, T.R., Stuart, A.E.A., Bernasconi, P., Stauffacher, M., Auer, J.A.,

599 Weishaupt, M.A., (2004) Assessment of mental stress in warmblood horses: heart

600 rate variability in comparison to heart rate and selected behavioural parameters.

601 Applied Animal Behavioural Science. 88, 121-136.

602

603 Schmidt, A., Mostl, E., Wehnert, C., Aurich, J., Muller, J., Aurich, C., (2010) Cortisol

604 release and heart rate variability in horses during road transport. Hormones and

605 Behaviour. 57(2), 209-215.

606 
607 Seaman, S.C., Davidson, H.P.B., Waran, N.K., (2002) How reliable is temperament

608 assessment in the domestic horse (Equus caballus)? Applied Animal Behaviour

609 Science. 78, 175-191.

610

611 Smith, T.E., French, J.A., (1997) Psychosocial stress and urinary cortisol excretion in

612 marmoset monkeys (Callithrix Kuhli). Physiology and Behaviour. 62, 225-232.

613

614 Stegman G.F., Jones, R.S (1998) Perioperative plasma cortisol concentration in the

615 horse. Journal of the South African Veterinary Assocation. 69(4), 131-142.

616

617 Strand, S.C., Tiefenbacher, S., Haskell, M., Hosmer, T., McDonnell, S.M., Freeman,

618 D.A., (2002) Behaviour and physiological responses of mares to short-term isolation.

619 Applied Animal Behaviour Science. 78, 145-157.

620

621 Toutain, P.L, Lassourd, V, Popot, M.A, Laroute, V, Alunerie, M., Bonnaire, Y., (1995)

622 Urinary cortisol excretion in the resting and exercising horse. Equine Veterinary

623 Journal Supplement. 18, 457-462.

624

625 Visser, E.K., van Reenen, C.G., Hopster, H., Schilder, M.B.H., Knaap, J.H.,

626 Barneveld, A., Blokhuis, H.J., (2001) Quantifying aspects of young horses'

627 temperament: consistency of behavioural variables. Applied Animal Behaviour

628 Science. 74, 241-258.

629

630 Visser, E.K., Ellis, A.D., van Reenen, C.G., (2008) The effects of two different

631 housing conditions on the welfare of young horses stabled for the first time. Applied

632 Animal Behaviour Science. 114, 521-533. 
634 Visser, K., Karlas, K., van Deurzen, I., Workel, van Reenen, K., (2010) Experts'

635 assessment of temperament in sport horse. Journal of Veterinary Behaviour. 5(4),

$636 \quad 214-215$.

637

638 Von Borell, E., Langbein, J., Despres, G., Hansen, S., Leterrier, C., Marchant-Forde,

639 J., Marchant-Forde, R., Minero, M., Mohr, E., Prunier, A., Valance, D., Veissier, I.,

640 (2007) Heart rate variability as a measure of autonomic regulation of cardiac activity

641 for assessing stress and welfare in farm animals - A review. Physiology \& Behaviour.

$64292,293-316$.

643

644 Wemelsfelder, F., Hunter, E.A., Mendl, M.T., Lawence, A.B., (2000) The

645 spontaneous qualitative assessment of behavioural expressions in pigs: first

646 explorations of a novel methology for integrative animal welfare measurement.

647 Applied Animal Behaviour Science. 67, 193-215.

648

649 Wemslefelder, F., Hunter, T.E.A., Mendl, M.T., Lawence, A.B., (2001) Assessing the

650 'whole animal': a free choice profiling approach. Animal Behaviour. 62, 209-220.

651

652 Winskill, L.C., Waran, N.K., Young, R.J., (1996) The effect of a foraging device (a

653 modified 'Edinburgh Foodball') on the behaviour of the stabled horse. Applied

654 Animal Behaviour Science. 48, 25-35.

655

656 Table and figure captions.

657

658 Table 1. Details of the subjects used in the study $(\mathrm{N}=32)$.

659

660 Table 2. The ethogram used for The Observer configuration. 
662 Table 3. Pattern matrix for PCA of the cortisol and duration of state behaviour

663 recorded during the four different routine husbandry procedures using oblimin

664 rotation of a three factor solution.

665

666 Table 4. A scale of behavioural indicators of stress in domestic stabled horses, as

667 revealed by principal component analysis (PCA) and behavioural assessment

668 completed by a professional panel.

669

670

671 Figure 1. Mean heart rate $(\mathrm{bpm}) \pm 1.0 \mathrm{SE}$ before (Baseline) and during the

672 husbandry procedures $(\mathrm{N}=18)$.

673

674 Figure 2. Mean salivary cortisol $(\mathrm{ng} / \mathrm{ml}) \pm 1.0 \mathrm{SE}$ before the husbandry procedures

675 (Baseline) and at peak following the procedure $(\mathrm{N}=19)$.

676 